\title{
Experimental Investigation of Using an Electromagnetic Gearbox to Ensure Dynamic Stability
}

\author{
Alexey Zhidkov ${ }^{1, *}$, Andrey Achitaev², and Elena Gracheva ${ }^{3}$ \\ ${ }^{1}$ Novosibirsk State Technical University, Department of Industrial Power Supply Systems, Russia \\ ${ }^{2}$ Siberian Federal University; Department of Hydropower Engineering and Electrical Networks, Russia \\ ${ }^{3}$ Kazan State Power Engineering University; Power supply of industrial enterprises, Russia
}

\begin{abstract}
Small generation is constantly increasing both in Russia and around the world. At the same time, more and more urgent problems arise related to the loss of dynamic stability of rotating machines with low inertia of their rotors. The paper proposes one of the variants for solving this problem by fundamental transformation of the principles of small generation, taking into account the development of a flexible coupling between a turbine and a generator by using an electromagnetic gearbox instead of a connecting coupling. Investigations are carried out on a physical prototype of an electromagnetic gearbox with observation of real parameters.
\end{abstract}

\section{Introduction}

There is a constant growth of small generation in Russia and in the world. According to the International Energy Agency, the share of small generation will be about $75 \%$ of the total volume of new energy generation until 2030, while more than $30 \mathrm{GW}$ of small generation is forecasted for Russia by 2035 [1].

At the same time, the implementation of small generation is always accompanied by the need to solve the problems of dynamic stability. At present, the main measure to ensure the dynamic stability of small generation is generator disconnection from the network. However, when the total volumes of small generation become significant, this solution will be unacceptable, since it will lead to a high power unbalance.

Despite the fact that many investigations are currently being carried out on ensuring the dynamic stability of small generation, this problem still does not have a universal solution. Firstly, this happens because the main amount of investigations is aimed at mitigating the consequences of dynamic instability, but not the reason. The efficiency of various methods of isolating small-power generators from the power system is assessed in [2-7]. However, due to the heterogeneity of power systems, these methods cannot be universal, therefore unique solutions should be developed for each specific case that significantly complicates the solution of the problem.

General approaches to the generator control using an absolute angle of the EMF vector are outlined in $[8,9]$. However, these studies were carried out for high-power machines, and the fundamental principles of implementing such systems for distributed generation are not determined there.
Electromagnetic couplings, gearboxes, continuously variable transmissions (CVT) are currently widely used in the field of power engineering for studying operating conditions of wind turbines (WT) [10-12]. The investigation of controllable flexible coupling of a turbine and a generator for a micro hydro was also performed [13]. But all these studies do not consider the problems of dynamic stability of synchronous generators operated on conventional fuel.

The investigation on the use of an electromagnetic CVT in a gas reciprocating power plant to ensure dynamic stability, which is based on mathematical modeling, was carried out in [14]. This study has shown that the use of an electromagnetic CVT is efficient for ensuring dynamic stability of a gas reciprocating unit (GRU) in case of emergency disturbances in the network.

The present paper continues the previous investigation with mathematical modeling carried out in [14] and presents the results of the new investigation on the efficiency of using an electromagnetic flexible coupling between a motor/turbine and a generator to ensure dynamic stability of small-power generating equipment using a physical model.

\section{Theoretical Background of Investigation}

A detailed description of theoretical foundations and the analysis of mathematical modeling are given in the paper where the previously performed investigation is described [14]. For understanding, only the main ideas of the previous study are given below.

An electromagnetic CVT, an electromagnetic gearbox (EG) or an electromagnetic coupling (EC) are

\footnotetext{
* Corresponding author: Lexxa1982@ngs.ru
} 
used as a damper during transient processes. The principle of their operation is illustrated in Fig. 1 and Fig. 2.
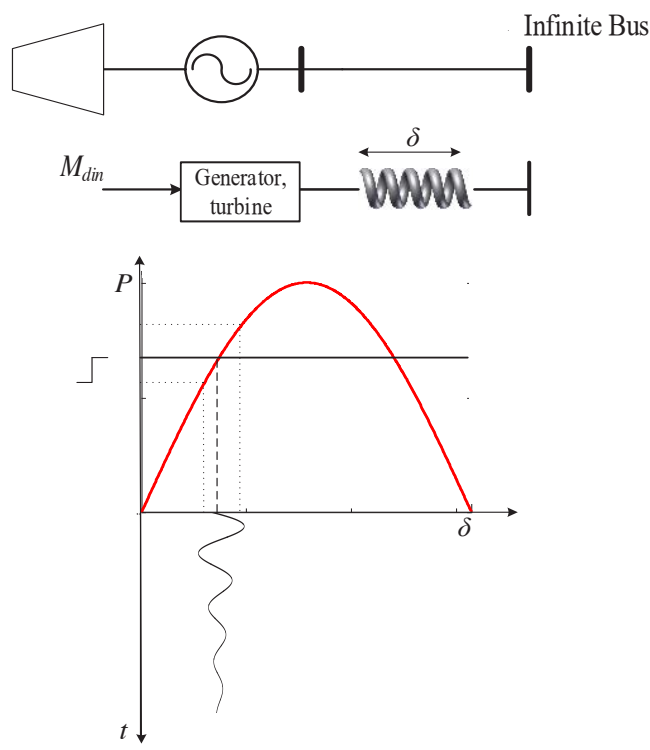

Fig. 1. Illustration of transient process for the conventional design.

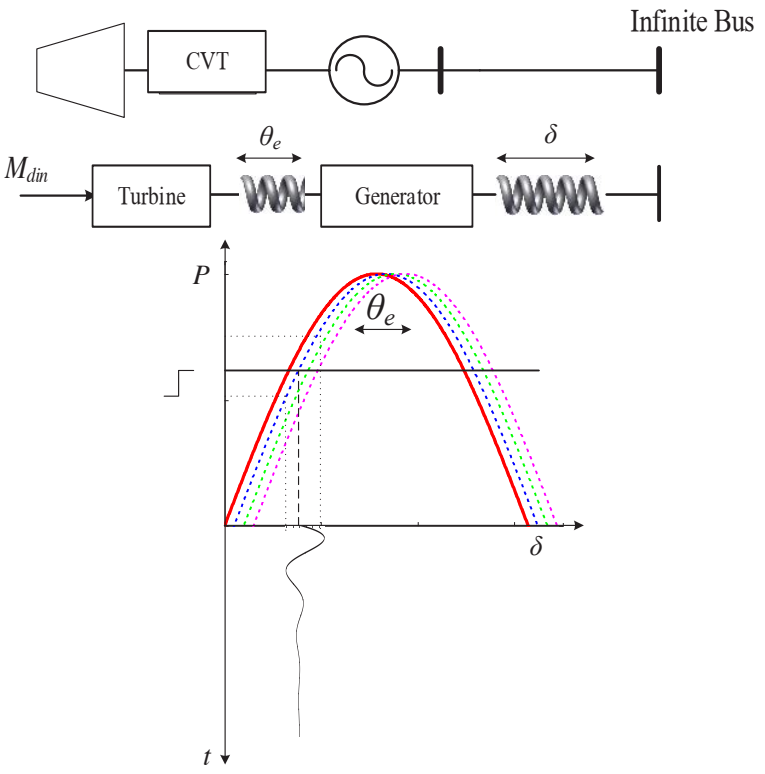

Fig. 2. Illustration of transient process for the use of an electromagnetic CVT.

The figures show the characteristics of a transient process for the conventional design and for the design with an electromagnetic CVT. The conventional design has one oscillating element, while the design with an electromagnetic CVT includes two oscillating elements. Therefore, the acceleration of oscillatory process damping can be observed when using an electromagnetic CVT.

The circuit of the investigated network is shown in Fig. 3. The analysis of transient processes in this network were carried out using Matab Simulink ${ }^{\circledR}$. As a result, the oscillograms of the rotation speed and angle of the GRU rotor under the investigated disturbance in the network are shown in Fig. 4 and Fig. 5.

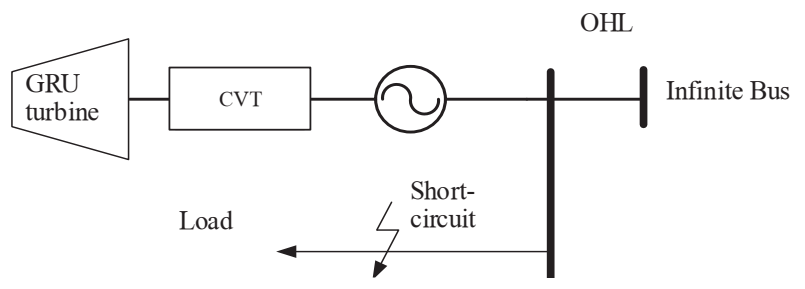

Fig. 3. Structure diagram of the investigated network.

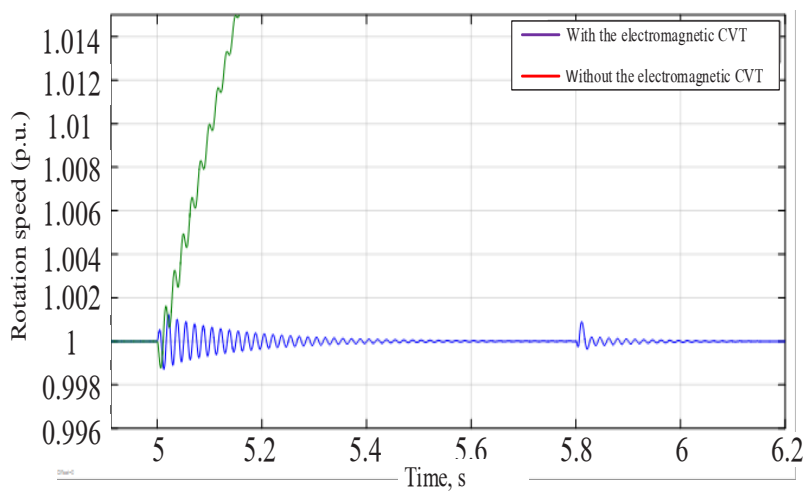

Fig. 4. Oscillograms of the rotation speed of the GRU rotor.

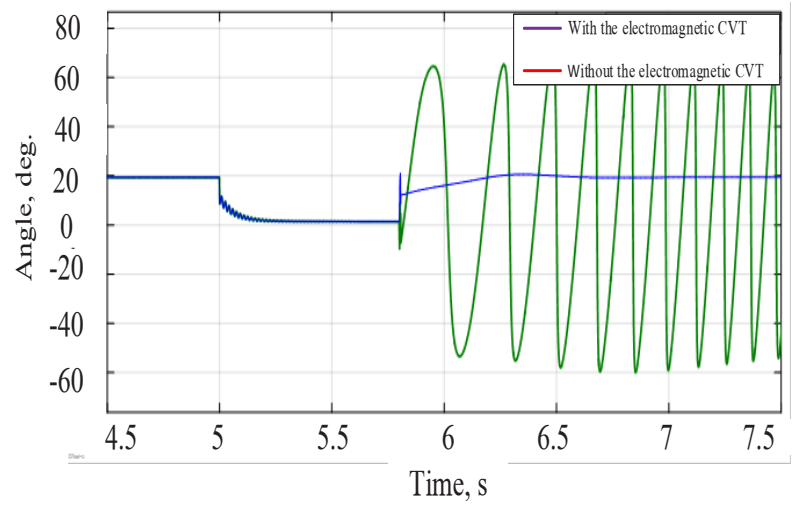

Fig. 5. Oscillograms of the relative angle of the GRU generator.

Based on this information given in the previously performed investigation [14], a preliminary conclusion was made that the use of an electromagnetic CVT is efficient to ensure dynamic stability. The purpose of this study is to confirm this conclusion on the physical model.

\section{Materials and methods}

To assess the influence of the EG on the dynamic stability of generating equipment, a prototype and a stand for investigating its characteristics were developed. The prototype was developed on the basis of previous studies and the results of similar investigations in related fields [14-18]. 
The appearance of the prototype in the assembled and disassembled form is shown in Fig. 6.
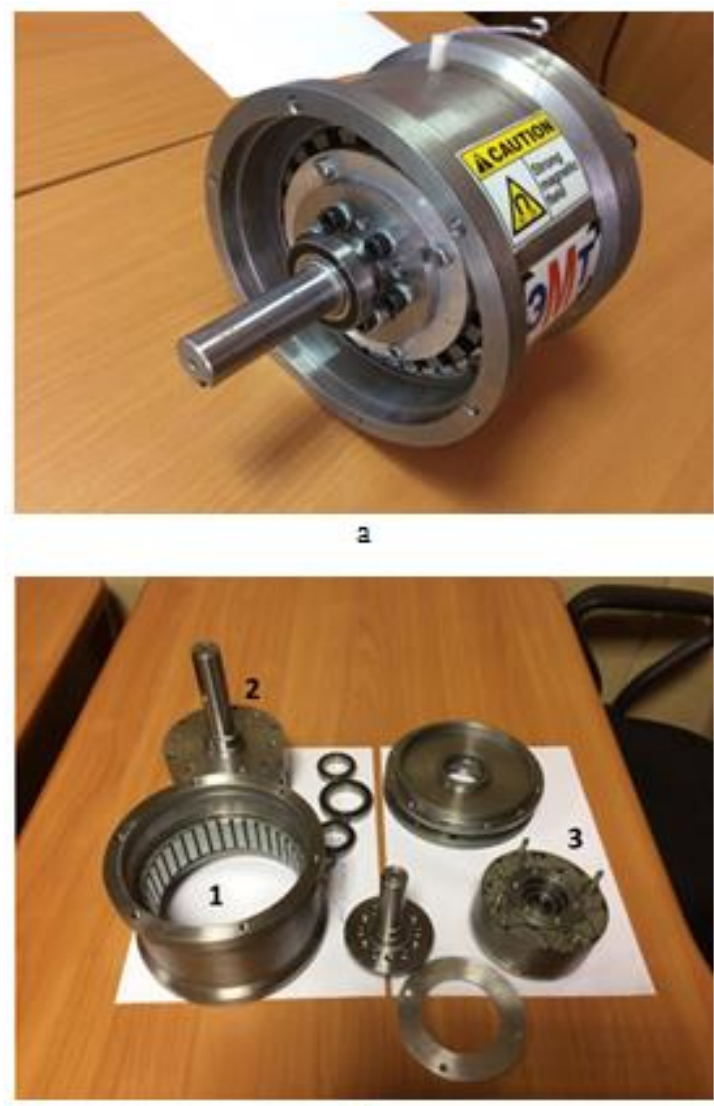

b

Fig. 6. The prototype: $a-$ assembled; $b$ - disassembled ( $1-$ stator, 2 - modulator (inner rotor), 3 - outer rotor).

The stator is made of a laminated magnetic core with a housing. Permanent magnets are placed in the stator. The modulator is made of steel and used as an input rotor. The outer rotor is made of a laminated magnetic core as the stator. $\mathrm{NdFeB}$ is used as the material of the permanent magnets. An analysis of the geometry and parameters of the magnetic system is presented in [12, 13].

The stand for investigation is shown in Fig. 7.

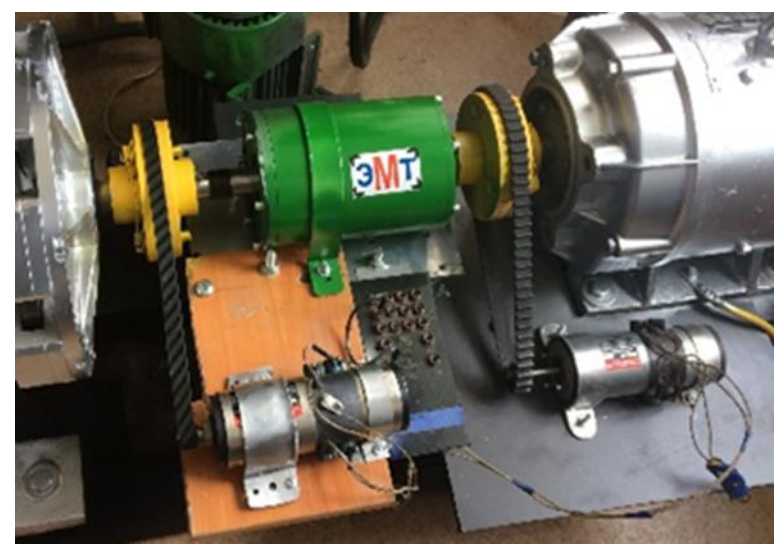

Fig. 7. The stand for investigating the prototype.

The transient process was recorded using oscilloscopes that control the output voltage of tachogenerators, which measure the rotation speed of each rotor of the prototype. A $5 \mathrm{~kW}$ motor simulating a gas turbine was installed at the input of the prototype. A $3 \mathrm{~kW}$ dynamic brake installed at the output was used to simulate operation of the electrical network.

\section{Results}

Fig. 8 shows the oscillogram of starting of the prototype under no load conditions.

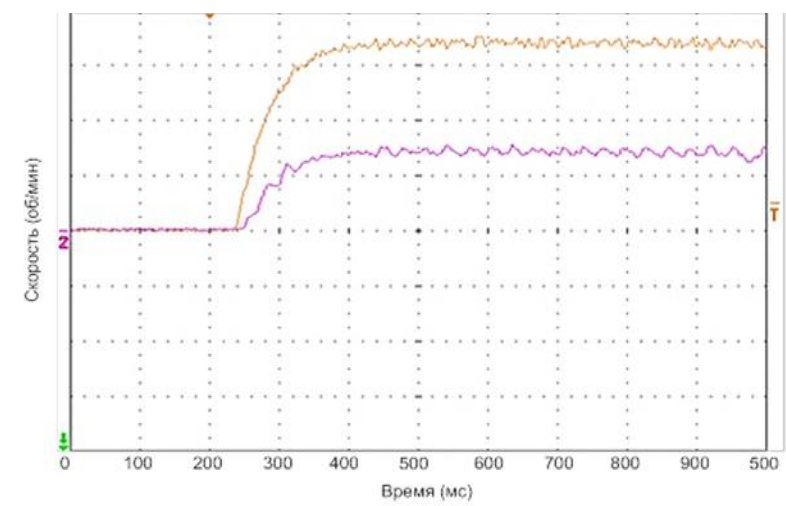

Fig. 8. Oscillogram of starting under no load conditions (red line - output, yellow line -input.

Fig. 9 shows the oscillograms of transient processes under various disturbances at the prototype output. Disturbances were simulated using the dynamic brake. 


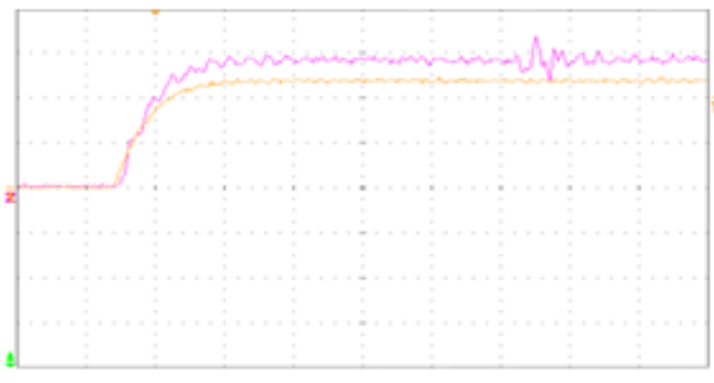

a
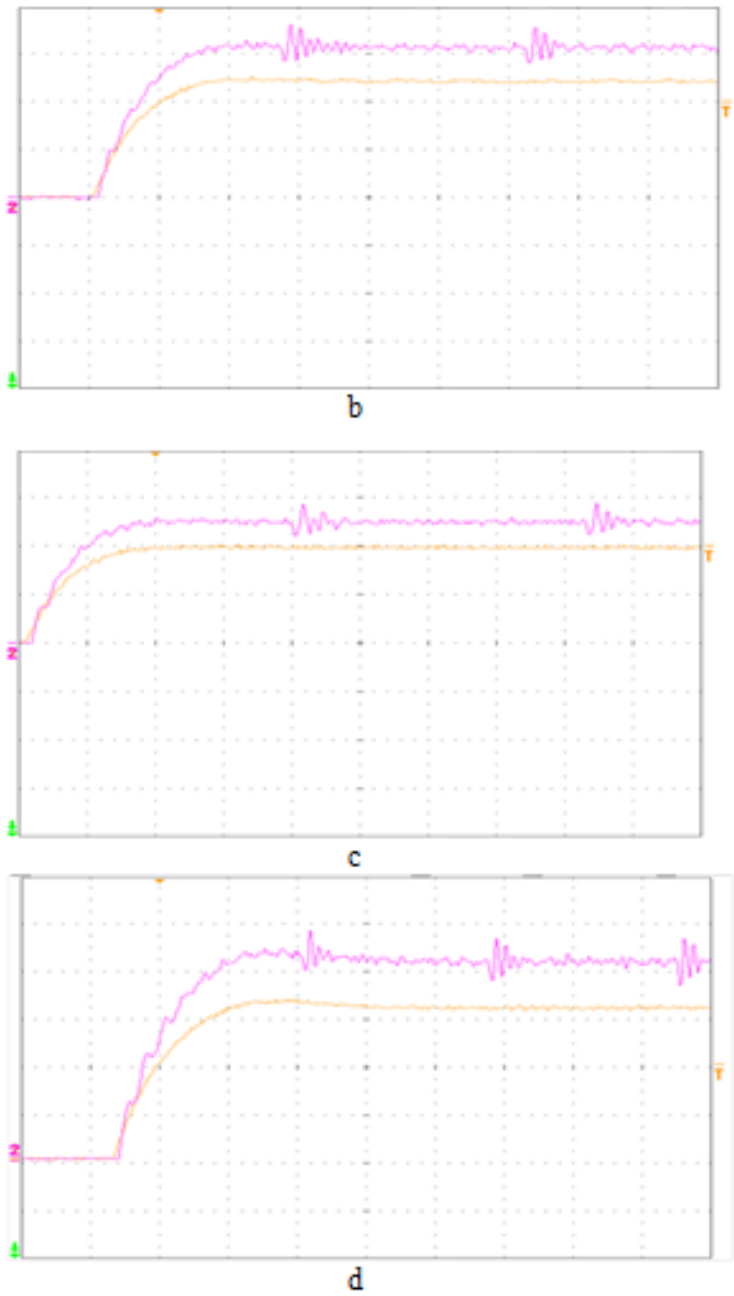

Fig. 9. Oscillograms of transient processes for the prototype under simulation of various disturbances in the network (yellow line - input, red line - output).

\section{Discussion}

The obtained oscillograms illustrate the influence of elastic magnetic interaction and show the efficiency of disturbances damping in the network by the prototype. The oscillograms also show that the oscillations at the prototype output are completely smoothed and not transmitted to its input. For the case of using the EG as a part of a generator unit, this means that oscillations in the network will have a significantly smaller influence on the operating conditions of a motor or turbine that will ultimately lead to enhancement of dynamic stability of the generating equipment.

\section{Conclusion}

The study of transient processes of generating units with flexible coupling between a motor/turbine and a generator using an electromagnetic gearbox instead of a mechanical gearbox under strong disturbances in the power system showed that the use of the electromagnetic gearbox can improve the dynamic stability of generating equipment. Therefore, the electromagnetic gearbox can be considered to be an efficient technical solution that ensures dynamic stability of generating equipment under fault disturbances in the network.

The reported study was supported by RFBR, research project No. 19-38-90034.

\section{References}

1. https://energy.skolkovo.ru/downloads/documents/SE neC/Research/SKOLKOVO_EneC_DER3.0_2018.02.01.pdf. (In Russian)

2. Ismoilov S.T., Trufakin S.S., Fishov A.G., "Multiagent voltage regulation in electrical networks with distributed generation and active consumers," Modern directions for development of relay protection and automation of power systems: Proceedings of the 4th International scientific and practical conference, Yekaterinburg: Publishing of Russian National Committee of CIGRE, 2013, pp. 99-100. (In Russian)

3. Arshad Saleem, Morten Lind, Manuela M. Veloso, "Multiagent based protection and control in decentralized electric power systems," 9th International Conference on Autonomous Agents and Multiagent Systems AAMAS, 2010.

4. Jignesh M. Solanki, Sarika Khushalani Solanki, Noel Schulz, "Multi-agent-based reconfiguration for restoration of distribution systems with distributed generators," Integrated Computer-Aided Engineering, 2010, No.17, pp. 331-346.

5. Jignesh M. Solanki, Sarika Khushalani, Noel N. Schulz, "A Multi-Agent Solution to Distribution Systems Restoration," IEEE transactions on Power Systems, 2007, No. 22 (3), pp. 1026-1034.

6. McArthur, S. D. J. and Davidson, E. M. and Catterson, V. M. and Dimeas, A. L. and Hatziargyriou, N. D. Ponci, F. Funabashi, T., "Multi-agent systems for power engineering applications - Part 2: technologies, standards and tools for building multi-agent systems," IEEE Transactions on Power Systems, 2007, No. 22 (4), pp. 1753-1759.

7. Alexandra von Meier, David Culler, Alex McEachern. Micro-Synchrophasors for Distribution Systems//Draft (version April 19, 2013).

8. Andreyuk V.A., "Management of the transient states of a power system using information on absolute voltage angle," Proceedings of the Scientific and Technical Center of the Unified Power System, 2011, No. 65, pp. 27-42. (In Russian) 
9. Andreyuk V.A., Asanbaev Yu.A., Skazyvaeva N.S., "Dynamic stability of the power system in the case of regulating power of generator turbines by an absolute angle," Moscow: Power Stations Journal, 2005, No. 12, pp. 11-16. (In Russian)

10. Aho J.P., Kraft L.G., "Control of a Wind Turbine with a Magnetic Continuously Variable Transmission for Mitigation of Torque Variations," Proc. of the 2011 AIAA/ASME Wind Symposium, USA, Orlando, 2011.

11. Polinder H., "Trends in Wind Turbine Generator Systems," IEEE Journal of emerging and selected topics in power electronics, 2013 September, V. 1, No. 3, pp. 174-185.

12. Udalov S. N., Pristup A. G., Achitaev A. A., "Research of magnetic transmission with variable gear ratio in a wind-driven generator for improving dynamic stability stoke," Bulletin of the Tomsk Polytechnic University - Geo Assets Engineering, 2015, vol. 326, No. 10, pp. 123-134.

13. Achitaev A.A., Zhidkov A.A., Mitrofanov S.V., Rusina A.G., "Investigation of controllable flexible coupling of a turbine and a generator of a micro hydro in an autonomous power system," Elektrichestvo Journal, 2020, No 1, pp. 25-31 (in Russian).

14. Zhidkov A.A. Using Electromagnetic Continuously Variable Transmission in Gas Reciprocating Power Plant to Ensure Dynamic Stability // 2020 International Conference on Industrial Engineering, Applications and Manufacturing (ICIEAM), Sochi, Russia, 18-22 May 2020, pp. 1-6.

15. Udalov S. N., Achitaev A. A., Pristup A. G., Bochenkov B. M., Pankratz Y. \& Tarbill R. D., "Increasing the regulating ability of a wind turbine in a local power system using magnetic continuous variable transmission," Wind Engineering, 2018, vol. 42, No. 5, pp. 411-435.

16. Montague R., Bingham C., Atallah K., "Servo control of magnetic gears," IEEE/Asme Transactions on Mechatronics, 2011, vol. 17, No. 2, pp. 269-278.

17. Dalpian G. M. et al., "Phenomenological band structure model of magnetic coupling in semiconductors," Solid state communications, 2006, vol. 138, No. 7, pp. 353-358.

18. A. V. Sapsalev, A. A. Achitaev, V. V. Bogdanov, N. P. Savin and O. B. Davydenko, "Structural model of a magnetic coupling," 2016 17th International Conference of Young Specialists on Micro/Nanotechnologies and Electron Devices (EDM), Erlagol, 2016, pp. 555-558. 